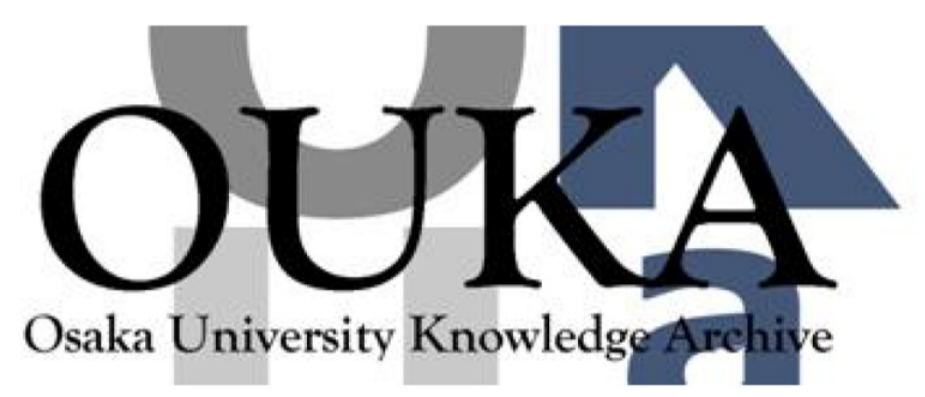

\begin{tabular}{|c|l|}
\hline Title & $\begin{array}{l}\text { Atomic-scale insights into the origin of } \\
\text { rectangular lattice in nanographene probed by } \\
\text { scanning tunneling microscopy }\end{array}$ \\
\hline Author(s) & $\begin{array}{l}\text { Li, Junhuan; Li, Shaoxian; Higashi, Tomoki et } \\
\text { al. }\end{array}$ \\
\hline Citation & $\begin{array}{l}\text { Physical Review B. 103(24) p. 245433-1-p. 245433- } \\
9\end{array}$ \\
\hline Issue Date & $2021-06-24$ \\
\hline oaire:version & VoR \\
\hline URL & https://hdl. handle. net/11094/83924 \\
\hline rights & $\begin{array}{l}\text { Copyright } 2021 \text { by the American Physical } \\
\text { Society. }\end{array}$ \\
\hline Note & \\
\hline
\end{tabular}

Osaka University Knowledge Archive : OUKA

https://ir. Library. osaka-u. ac. jp/

Osaka University 


\title{
Atomic-scale insights into the origin of rectangular lattice in nanographene probed by scanning tunneling microscopy
}

\author{
Junhuan Li, ${ }^{1}$ Shaoxian Li $\odot,{ }^{1, *}$ Tomoki Higashi, ${ }^{2}$ Kentaro Kawai $\odot,{ }^{1}$ Kouji Inagaki $\odot,{ }^{1}$ \\ Kazuya Yamamura $\bullet^{1,3}$ and Kenta Arima ${ }^{1, \dagger}$ \\ ${ }^{1}$ Department of Precision Engineering, Graduate School of Engineering, Osaka University, 2-1, Yamada-oka, Suita, Osaka 565-0871, Japan \\ ${ }^{2}$ Division of Applied Science, School of Engineering, Osaka University, 2-1, Yamada-oka, Suita, Osaka 565-0871, Japan \\ ${ }^{3}$ Research Center for Precision Engineering, Graduate School of Engineering, Osaka University, 2-1, Yamada-oka, \\ Suita, Osaka 565-0871, Japan
}

(Received 29 March 2021; revised 8 June 2021; accepted 10 June 2021; published 24 June 2021)

\begin{abstract}
We conducted atomic-scale scanning tunneling microscopy of a graphene nanosheet on graphite. In addition to a rhombus lattice representing the $(\sqrt{3} \times \sqrt{3}) R 30^{\circ}$ superstructure, we resolved another quadrangle lattice similar to a rectangle in the sheet. Its lattice size was approximately $0.37 \times 0.22 \mathrm{~nm}^{2}$. To clarify the origin of this unique rectangular lattice, the overlap of the $\sqrt{3} \times \sqrt{3}$ superstructures along the direction of their long diagonals was theoretically examined using a simple model. The electron distribution with high energy in the occupied states of armchair-edged graphene nanoribbons (AGNRs) was calculated based on first principles. A rectangular lattice, resembling the one observed experimentally, was found to form on the AGNR under a specific width condition. This finding was also analyzed in terms of Clar's theory and the scattering of electron waves. We propose that wrinkles and adsorbates in graphene play a role similar to an armchair edge, resulting in the $\sqrt{3} \times \sqrt{3}$ phase. If these local defects are in close proximity, the rhombus phases interact to generate electronic structures predicted for AGNRs. This is probably the reason why a rectangular lattice was imaged on the graphene sheet that is not an ideal AGNR.
\end{abstract}

DOI: 10.1103/PhysRevB.103.245433

\section{INTRODUCTION}

Graphene is an intensively studied material because of its various unique properties [1-3]. For example, the electron motion in graphene is governed by the Dirac equation with vanishing rest mass [4,5], which provides promising potential of graphene for its use in future electronic materials [6,7]. Another important trend in graphene research is the control of its topological defects. Special emphasis has been made to unveil the relationship between the electronic or chemical properties of graphene-based materials and local atomic arrangement. For example, point defects and dislocations on graphene were revealed to provide more efficient anchor sites to directly couple adatoms, such as transition metal atoms, leading to high chemical reactivity $[8,9]$. Recent papers have suggested that heteroatom-doping in carbon could result in active sites for the oxygen reduction reaction [10,11]. These defects give rise to a large increase in the local density of states (LDOS) near the Fermi level $\left(\varepsilon_{\mathrm{F}}\right)$ [12].

Among its many types of structural defects, most studies have investigated the edges of nanosized graphene sheets (nanographene) or semi-infinite sheets [13-16]. In particular, the nonbonding $\pi$-electron state, topologically originated from the edge structure, is important. According to prior

\footnotetext{
*Present address: Institute of Chemistry and Chemical Engineering, Ecole Polytechnique Fédérale de Lausanne (EPFL).

${ }^{\dagger}$ Corresponding author: arima@prec.eng.osaka-u.ac.jp
}

studies [17-21], this edge state appears around $\varepsilon_{\mathrm{F}}$ in the case of zigzag edges, which has attractive electronic, spintronic, and catalytic features [22-24]. On the other hand, nanographene with armchair edges or armchair-edged graphene nanoribbon (AGNR), has an advantageous tunable bandgap $\left(E_{\mathrm{g}}\right)$. Pioneering works by Nakada et al. presented tight-binding band calculations of AGNRs to unveil that the width of AGNRs critically controls whether the system is metallic or semiconducting [25]. Additionally, the energy dispersion of atomically precise AGNRs has been investigated by scanning tunneling spectroscopy on Au [26], which agreed with theoretical simulations in the same system [27].

In addition, the edge plays the role of a potential barrier that interferes with electron waves. This electron wave scattering at edges is manifested as a superstructure pattern, which can be imaged through scanning tunneling microscopy (STM). Among various solid surfaces, including metals [28], this issue has been studied on graphite and its related nanomaterials over the last several decades. However, the relationship between an interference pattern and local atomic geometry, which is fundamental and important for characterizing edges, is still an area to be investigated. For example, the observations of long-range electronic perturbations caused by defects were first made on a graphite surface through STM in 1988 [29]. These oscillations had a characteristic wavelength $3 / 2$ times that of the graphite lattice and are called $(\sqrt{3} \times \sqrt{3}) R 30^{\circ}$ superstructures. Some experiments have insisted that the $\sqrt{3} \times \sqrt{3}$ phase appears only near an armchair edge on a stepped graphite surface [30,31], which is consistent with 
theoretical simulation [32]. Other researchers have reported a coexistence of this phase and honeycomb superstructures near an armchair edge $[33,34]$. Even on graphene nanoribbons, one research group resolved a spatially uniform $(\sqrt{3} \times \sqrt{3}) R 30^{\circ}$ superstructure while another study found fluctuations in its periodicity $[14,35]$. These discrepancies in experiments may be caused by the structural disorder of armchair edges and other local defects, such as vacancies and extrinsic adsorbates incorporated inside the honeycomb lattice, that are likely to alter intrinsic electronic properties in graphene [36-39]. Understanding the superstructure pattern is expected to reveal the role of edges and other defects in generating unique electronic structures. This understanding will also provide guidelines on how to modify a graphene network to exhibit specific electronic, spintronic, and catalytic functions, which will be vital to the future development of graphene-related science and technology.

We performed STM experiments on a small graphene sheet on graphite. Together with a $(\sqrt{3} \times \sqrt{3}) R 30^{\circ}$ phase, we resolved a rectangular superstructure whose origin is the main focus in this study. To examine if the rectangular lattice is formed by the superposition of two $\sqrt{3} \times \sqrt{3}$ phases at a different phase, we modeled AGNRs and conducted first-principles calculations. Simulated STM images revealed the conditions of a ribbon width and sample bias to create a rectangular phase quite similar to that observed experimentally. Then we discussed an imaging mechanism explaining why the rectangular lattice simulated on AGNRs appeared on nanographene which was not an ideal nanoribbon with parallel armchair edges.

\section{METHODOLOGY}

\section{A. Experimental method}

STM experiments were performed on a commercial, highly oriented pyrolytic graphite (HOPG) sample. A fresh HOPG surface was created by cleaving the HOPG sample with adhesive tape in ambient air. After cleaving, the sample was immediately transferred to an ultrahigh vacuum (UHV: $\sim 2 \times 10^{-8} \mathrm{~Pa}$ ) chamber for STM observations. Neither thermal treatments nor baking was applied to the HOPG surface prior to observations. We used Pt/Ir tips that were mechanically cut and then cleaned by electron bombardment. Images were taken at room temperature in a constant current mode using a commercial UHV STM system (JEOL, JSTM-4500XT).

To confirm superstructure patterns on HOPG, similar STM experiments were conducted using another nanographene, i.e., reduced graphene oxide ( $\mathrm{rGO}$ ). The rGO flakes were obtained by the chemical reduction of GO with hydrazine. The preparation of rGO flakes is described elsewhere in detail [40,41]. A droplet of an rGO solution was put on the cleaved HOPG substrate, which was followed by spin coating and drying by blowing $\mathrm{N}_{2}$ gas to form dispersed rGO flakes.

\section{B. Simulation theory}

All first-principles calculations based on density functional theory were performed using STATE code with ultrasoft pseudopotentials $[42,43]$. We used the PerdewBurke-Ernzerhof generalized-gradient approximation as the

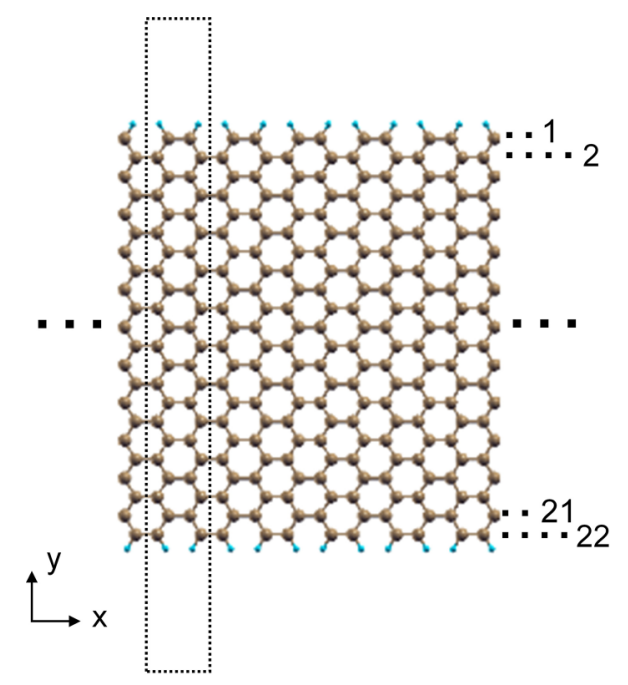

FIG. 1. Schematic of the AGNR model for simulations. The edge dangling $\sigma$ bonds are passivated by hydrogen atoms (small balls). The dashed frame indicates the supercell used in the calculation.

exchange-correlation functional [44]. A plane-wave basis set was used to expand wave functions and charge density with cutoff energies of 36 and $400 \mathrm{Ry}$, respectively. We first optimized the lattice constant of an infinite graphene sheet. Brillouin zone integration was done with a uniform grid of $24 \times 24 k$ points. The obtained lattice constant was $2.466 \AA$, which is in good agreement with experiment [45]. These results were used to construct the initial atomic coordinates of a supercell for AGNR.

A schematic model of AGNR with a width of $W=22$ used in this article is shown in Fig. 1 as an example. In terms of ribbon width, $W$ is defined as the number of dimer lines across the ribbon width. The armchair edges of the ribbons in this study were treated as single hydrogenated. In Fig. 1, the size of the supercell along the $x$ and $y$ directions is 4.26 and $43.65 \AA$, and vacuum distances of 15.90 and $10.60 \AA$ separate the ribbons in plane and between planes, respectively. The Brillouin zone integration was done with a uniform grid of $30 k$ points along the edge direction. The atomic positions in the cell were optimized to relax until all the forces on the nuclear coordinates were below a threshold of $2.6 \times 10^{-3} \mathrm{eV}^{-1}$.

STM images were simulated using the Tersoff-Hamann approximation [46]. In this approach, the local tunneling current between sample and probe is proportional to the sum of the electron density of orbitals in the interval $\left[\varepsilon_{\mathrm{F}}+e V_{\mathrm{s}}, \varepsilon_{\mathrm{F}}\right]$ for a negative sample bias $\left(V_{\mathrm{s}}\right)$. Here $e$ and $\varepsilon_{\mathrm{F}}$ denote the elementary charge $(e>0)$ and the Fermi energy, respectively. The electrons tunnel from the occupied states of the sample to the empty states of the probe in this condition. Our $V_{\mathrm{s}}$ in the experiments was low, ranging from -0.01 to $-0.05 \mathrm{~V}$. In this case, simulated STM images can be greatly affected by the position of $\varepsilon_{\mathrm{F}}$. To avoid inaccuracies in the $\varepsilon_{\mathrm{F}}$, we used the sum of the electron density of orbitals in $\left[\varepsilon_{\mathrm{HOMO}}+e V_{\mathrm{s}}, \varepsilon_{\mathrm{HOMO}}\right]$, where $\varepsilon_{\text {Hомо }}$ indicates the energy level of the highest occupied molecular orbital (HOMO). Computed LDOS contours $\left(6.72 \times 10^{-4} / \AA^{3}\right)$ were obtained for the simulated STM images at the corresponding $V_{\mathrm{s}}$. 
(a)

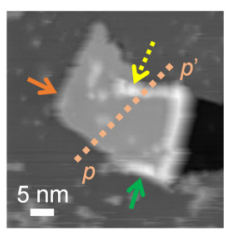

(b)

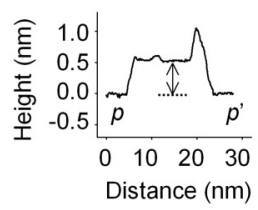

(c)

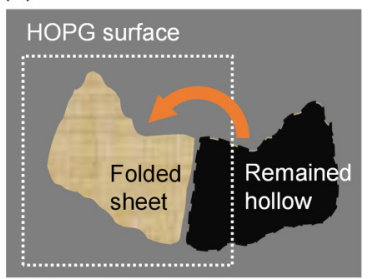

(d)

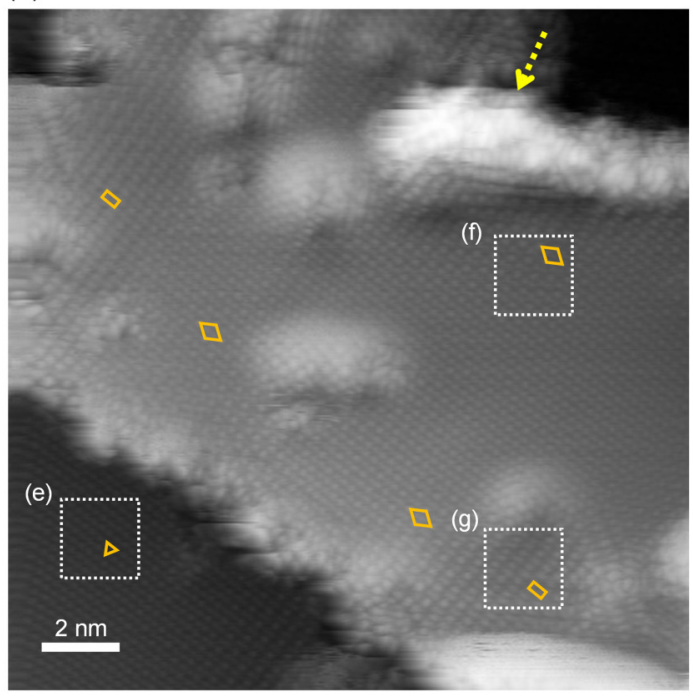

(e)

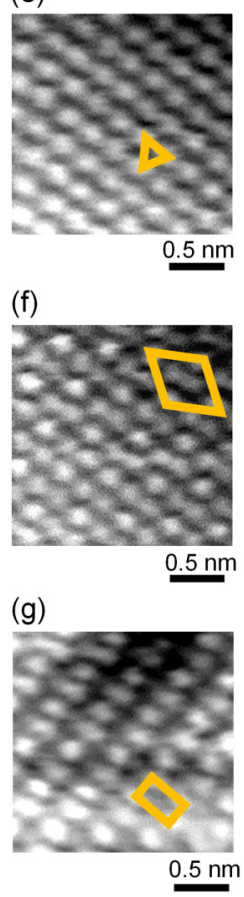

FIG. 2. (a) STM image of a small graphene sheet on HOPG. The solid arrows pointing toward an outer edge or a protrusion are placed on the image for later discussion. (b) Height profile along $p-p^{\prime}$ in (a). (c) Schematic depiction of the graphene sheet corresponding to (a); the dotted area indicates the region imaged in (a). (d) Magnified STM image of the folded sheet in (a). The dotted arrows in (a) and (d) indicate the same position. (e)-(g) Typical distributions of bright dots in (d). The unit cells of (e) triangular, (f) rhombohedral, and (g) rectangular lattices are superimposed in each image.

\section{RESULTS AND DISCUSSION}

Figure 2(a) shows an STM image of a small graphene sheet on HOPG. $V_{\mathrm{s}}$ and tunneling current $\left(I_{\mathrm{t}}\right)$ were set to $-0.05 \mathrm{~V}$ and $0.2 \mathrm{nA}$, respectively. The cross-sectional profile along the $p-p$ ' line in Fig. 2(a) is provided in Fig. 2(b). Except for the right edge, the sheet thickness in Fig. 2(b) is measured to be approximately $0.5 \mathrm{~nm}$, as indicated by an arrow. At the right side of the sheet in Fig. 2(a), there exists a hollow. It is probable that the graphene sheet in Fig. 2(a) was partially exfoliated from the HOPG surface and then folded to leave a hollow at its right-hand side, as schematically depicted in Fig. 2(c). The measured thickness of the graphene sheet in Fig. 2(b) is larger than the theoretical value of $0.34 \mathrm{~nm}$, which corresponds to the monoatomic step height of a graphite surface. This is probably due to nonplanar deposition of the graphene sheet $[47,48]$, leading to the formation of wrinkles. Adsorbed molecules on graphene are also known to increase a measured thickness [49]. Note that there are two possible origins to form such a folded graphene sheet. One of the origins of formation is during the cleavage of the HOPG surface by adhesive tape. The other is the vertical displacement of the top graphene layer from the substrate mediated by the interaction of an STM tip with the HOPG sample [50]. In previous studies, such STM manipulation has been used for producing graphene nanoribbons [16,51,52]. In this work, we focus on the superstructures on the folded graphene sheet in Fig. 2(a).

Figure 2(d) shows a magnified STM image of the monolayer graphene sheet in Fig. 2(a). The image exhibits atomic- scale bright dots that form different patterns. Figures 2(e)2(g) show the typical three patterns extracted from Fig. 2(d) wherein the gray scale was adjusted to highlight atomic corrugations. On the HOPG terrace, there exist triangular lattices, as shown in Fig. 2(e). Bernal stacking is the most reliable hypothesis for their observation [53,54]. In this configuration, three carbon atoms are placed right above the hollow sites of the graphene layer below, which are denoted as $\beta$ sites. The distance between neighboring dots at the $\beta$ site is $0.25 \mathrm{~nm}$. On the folded sheet in Fig. 2(d), there exists a rhombus pattern, an example of which is shown in Fig. 2(f). For later discussion, the lengths of the long and short diagonals of this rhombus lattice were analyzed and determined to be $0.75 \pm$ 0.01 and $0.46 \pm 0.01 \mathrm{~nm}$, respectively. Considering this size, it is reasonable to assume that the rhombus represents the $(\sqrt{3} \times \sqrt{3}) R 30^{\circ}$ superstructure. This $\sqrt{3} \times \sqrt{3}$ phase has been reported by many groups on graphite surfaces near complete or defective armchair edges [33], as well as on graphene grown on $\mathrm{SiC}$ [55]. In addition, it is striking that another rectangular quadrangle lattice is clearly visible on the folded sheet, as shown in Fig. 2(g). This phase, which is hereafter referred to as a "rectangular lattice," was confirmed at different areas on the sheet; two examples are indicated in Fig. 2(d). The rectangular lattice has long and short sides with lengths of $0.37 \pm 0.02$ and $0.22 \pm 0.01 \mathrm{~nm}$, respectively. A notable feature in Fig. 2(g) is that the long-side direction of the lattice is the same as, or parallel to, that of the long diagonal of the $\sqrt{3} \times \sqrt{3}$ phase in Fig. 2(f). Another feature observed is that, in contrast to circular bright dots at the vertices of the $\sqrt{3} \times \sqrt{3}$ 
(a)

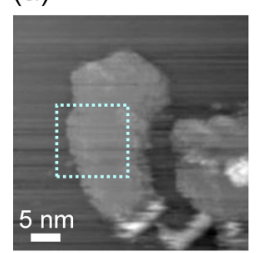

(b)

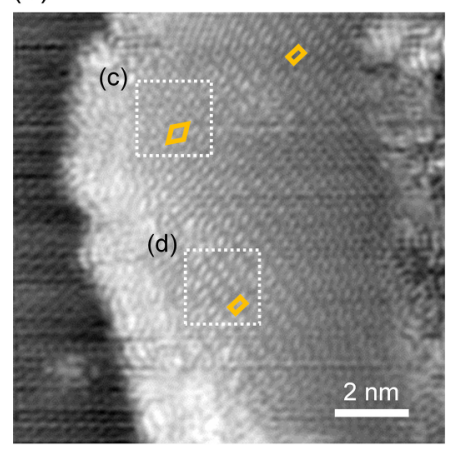

(c)

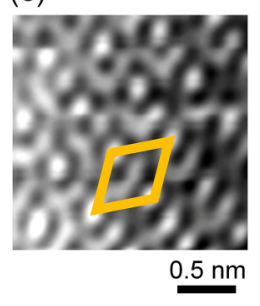

(d)

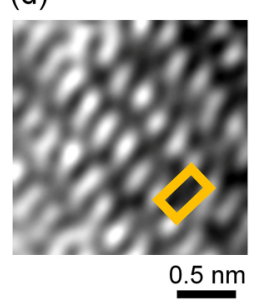

FIG. 3. (a) STM image of a HOPG surface loaded with a rGO sheet. (b) Enlarged STM image of the area indicated by the dotted line in (a). Dot patterns exhibiting (c) $\sqrt{3} \times \sqrt{3}$ and (d) rectangular lattices in (b).

phase in Fig. 2(f), those of the rectangular lattice tend to be elongated along its long-side direction in Fig. 2(g).

To investigate the origin of this rectangular lattice in Fig. 2(g), we first rule out the possibility of tip artifacts. Figure 3 shows an image on a rGO sheet dispersed on HOPG. $V_{\mathrm{s}}$ and $I_{\mathrm{t}}$ were set to $-0.01 \mathrm{~V}$ and $0.1 \mathrm{nA}$, respectively. In Fig. 3(a), a small flake exists, part of which is enlarged in Fig. 3(b). The atomic-scale corrugations of the flake in Fig. 3(b) are mainly composed of two superstructures. One has rhombus and the other has rectangular lattices, as shown in Figs. 3(c) and 3(d), respectively. The sizes of these lattices match those in Figs. 2(f) and 2(g). In addition, the direction of the long side of the lattice in Fig. 3(d) is parallel to that of the long diagonal in Fig. 3(c). The bright spots of the rectangular lattice are distorted along its long side. These features are similar to those in Fig. 2, which indicate that Fig. 2 is not a "ghost" image due to tip conditions but is derived from intrinsic electronic structure in the small graphene sheet.

A similar rectangular lattice to Fig. 2(g) has been observed on graphene-based material by other research groups [56,57]. However, except for one group [58], its imaging mechanism has not been discussed in detail. STM observations conducted on exfoliated GO sheets on HOPG reported by Pandey et al. revealed a unit cell with a size of $0.41 \times 0.27 \mathrm{~nm}^{2}$ over distances spanning a few nanometers. They insisted that the unit cell originated owing to oxygen functional groups bonded to carbon atoms in the GO sheet. Interestingly, our rectangular lattice in Fig. $2(\mathrm{~g})$ is similar to the shape reported in the literature. However, the size of our rectangular lattice is different, which indicates that probably our rectangular lattice is created by a mechanism other than the periodic distribution of oxygen functionalities. Regarding the size, it should be noted that the short-side length of the rectangular lattices in Fig. 2(g)

is almost half of the short diagonals of the $\sqrt{3} \times \sqrt{3}$ lattices in Fig. 2(f). Considering the aligned directions of the two lattices in Figs. 2(f) and 2(g), as mentioned in a former paragraph, a likely explanation for the creation of the rectangular lattice in Fig. $2(\mathrm{~g})$ is the overlap of two different $\sqrt{3} \times \sqrt{3}$ superstructures. Recently, another group presented a detailed STM study of a graphite surface with monoatomic armchair step edges [31]. They visualized a mixture of $(\sqrt{3} \times \sqrt{3}) R 30^{\circ}$ and honeycomb superstructures near structurally defective armchair edges. They claimed that the honeycomb pattern results from a lateral superposition of two sets of $(\sqrt{3} \times \sqrt{3}) R 30^{\circ}$ superstructures with different phases. Inspired by their work, we propose that the rectangular lattice in Fig. 2(g) is formed by another overlap or a superposition of two sets of the $\sqrt{3} \times \sqrt{3}$ phases along the direction of the long diagonals.

To examine this possibility in a simple model, we investigated the electronic structures of AGNR, which has armchair step edges in close proximity, by first-principles calculations. We first checked the validity of our simulations by investigating $E_{\mathrm{g}}$ as a function of an AGNR width based on its band structure. As shown in Fig. 4(a), it is clear that the plots can be divided into three subclasses, i.e., $W=3 a-2,3 a-1$, and $3 a$ wherein $a$ is an integer. For all subclasses, $E_{\mathrm{g}}$ decreases with increasing ribbon width and tends to zero. The graph indicates that the width and subclass of AGNR determine whether the system is metallic or semiconducting. This result in Fig. 4(a) is in good agreement with literature $[59,60]$.

We then simulated STM images at a $V_{\mathrm{s}}$ of $-0.05 \mathrm{~V}$ for AGNRs with the width ranging from $W=22$ to $W=30$. We found that STM images change periodically as the ribbon width increases. Figures 4(b)-4(d) summarize the results for AGNRs with $W=3 a-2,3 a-1$, and $3 a$, respectively. Figure 4(b) reveals that electron density is slightly high at the armchair edges. The dot patterns form a $(\sqrt{3} \times \sqrt{3}) R 30^{\circ}$ superstructure in the interior of the ribbons. In the middle of the ribbons, single cocoonlike spots occupy the vertices of the $\sqrt{3} \times \sqrt{3}$ cell. For nanoribbons with $W=3 a-1$ in Fig. 4(c), the corrugations at edges and near edges are similar to those in Fig. 4(b). A $\sqrt{3} \times \sqrt{3}$ lattice is formed in the entire area of the ribbons, however, a feature of single cocoonlike spots is absent. In contrast, for the case of $W=3 a$ in Fig. 4(d), electrons are not localized at edges and bright dots in the images appear to form a rectangular lattice. Details on the distribution and the shape of the bright spots as well as their relationship with the position of a carbon network will be discussed later. This periodic change of images was confirmed to continue until reaching a width of at least $W=61,63$, and 65 . The ribbon width for these $W$ values was approximately $7.9 \mathrm{~nm}$. Calculated $E_{\mathrm{g}}$ values for the ribbons with $W=61,63$, and 65 were $0.19,0.11$, and $0.03 \mathrm{eV}$, respectively.

Next, we address why STM images change periodically with every three increases of $W$ in Figs. 4(b)-4(d). We recall that in graphene nanoribbons, the occupied states of such a small $V_{\mathrm{S}}$ near $\varepsilon_{\mathrm{F}}$ belong to the $\pi$ electrons. Therefore, the STM signal essentially reflects the distribution of $\pi$ electrons over the lattice. Clar's theory can be used to describe the electronic states of $\pi$ electrons of $s p^{2}$-bonded materials [61], including graphene, qualitatively. In this theory, six delocalized $\pi$ electrons form a Clar sextet, representing the resonance of two complementary hexagonal Kekulé configu- 


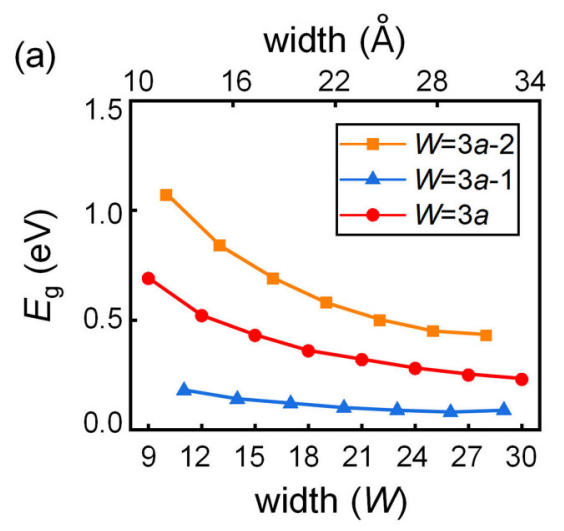

(b) $W=3 a-2$

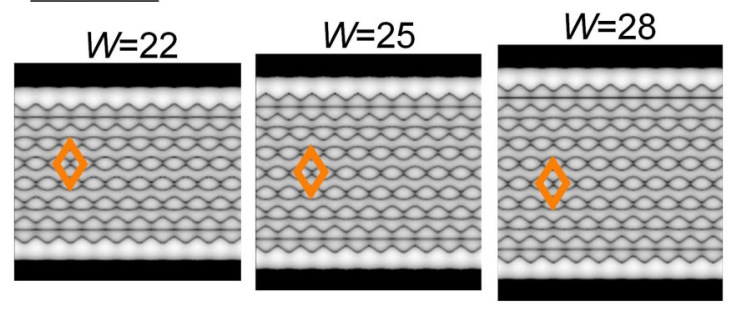

(c) $W=3 a-1$

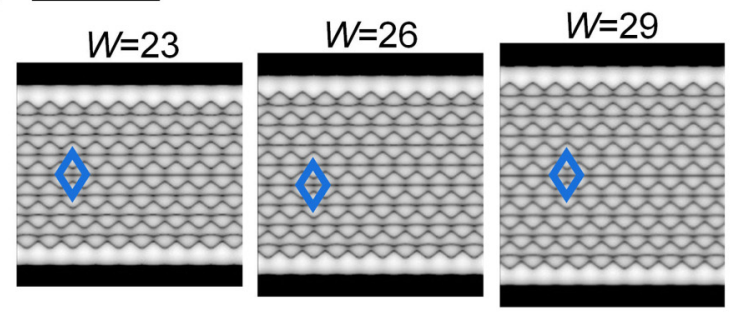

(d) $\quad W=3 a$

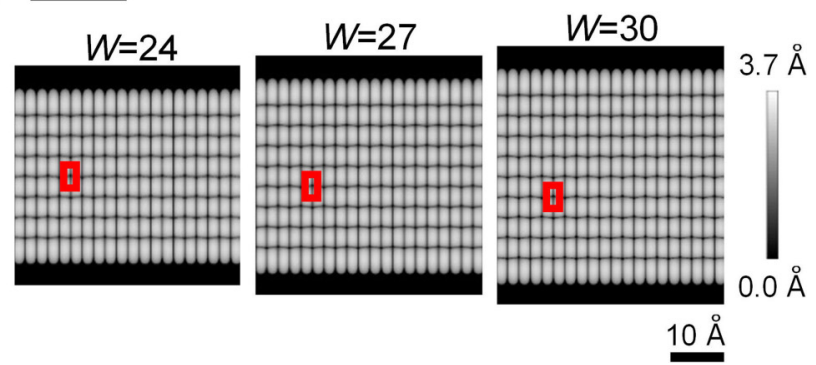

FIG. 4. (a) Calculated bandgaps $\left(E_{\mathrm{g}}\right)$ as a function of AGNR width. The filled square, triangular, and circular symbols represent data for an AGNR with $W=3 a-2,3 a-1$, and $3 a$, respectively. (b)-(d) Simulated STM images. A unit structure is superimposed in each image. (b) AGNRs with $W=22$ (left), $W=25$ (middle), and $W=28$ (right). AGNRs with (c) $W=23, W=26$, and $W=29$, and (d) $W=24, W=27$, and $W=30$.

rations with alternating single and double bonds. Figure S1(a) in the Supplemental Material (SM), depicts the resonance of electronic structures in benzene [62]. For a given material, the representation with a maximum number of Clar sextets is called the Clar formula. If the maximum number of Clar sextets is $n$, such a Clar bond configuration has the resonance (a)

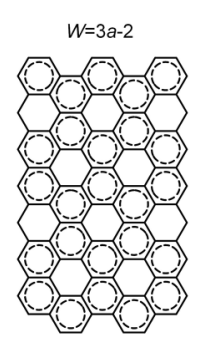

(b)

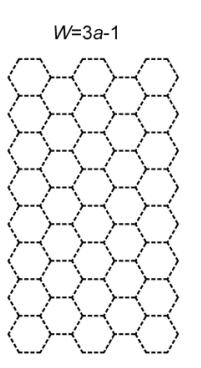

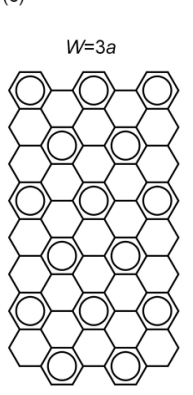

(d)

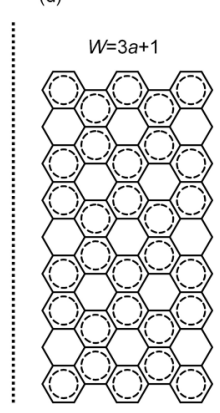

FIG. 5. Superpositions of all possible Clar formulas for the AGNRs. (a) A ribbon with a width index of $W=3 a-2$ has two equivalent Clar formulas. (b) A ribbon with a width index of $W=$ $3 a-1$ has more than two equivalent Clar formulas. The dotted lattice indicates the averaged result of all the possible formulas. (c) A ribbon with the width index of $W=3 a$, which shows one unique Clar formula. (d) A ribbon with a width index of $W=3 a+1$, which has a Clar formula pattern similar to that in (a).

of $2^{n}$ Kekulé-type bond formulas [63]. By taking account of all possible Kekulé-type bond formulas, we can best predict various properties of $s p^{2}$-bonded materials, ranging from the local density of $\pi$ states to bond lengths $[64,65]$. Thus, maximizing the number of Clar sextets unifies a maximum number of resonant Kekulé formulas in one single Clar representation.

As shown in Fig. S1(b), an infinite graphene sheet has three equivalent Clar formulas [62]. In these formulas, all $\pi$ electrons belong to a Clar sextet and the bonds sticking out of a Clar sextet are single bonds without any localized double bonds. As a result, the pattern of Clar sextets forms a $(\sqrt{3} \times \sqrt{3}) R 30^{\circ}$ superstructure in each Clar formula. The superposition of these three equivalent Clar formulas leads to a uniform $\pi$-electron distribution over the graphene sheet.

In the case of graphene nanoribbons, the uniform $\pi$ electron distribution of graphene can be disrupted due to edges. Even if we confine our focus on single-hydrogenated armchair edges, $\pi$ electrons are not allowed to form only Clar sextets but instead form a mixture of Clar sextets and localized double bonds in some ribbons, depending on ribbon width. Based on the study by Wassmann et al. [66], our AGNR can be divided into three subclasses according to the number of different equivalent Clar formulas. The simplest case is AGNR with $W=3 a$, which has only one unique Clar representation with a maximal number of Clar sextets for its unit cell. The next case is AGNR with $W=3 a-2$, in which there exist two equivalent Clar formulas. Finally, AGNR with $W=3 a-1$, have multiple, or more than two, Clar representations maximizing the number of Clar sextets. In the latter two cases, the electronic structure of each AGNR is determined by the coexistence of all possible Clar formulas. Figures 5(a)-5(d) depict the periodic change of the superposition of Clar formulas and explains why simulated STM images change periodically with every three increases of $W$ in Figs. 4(b)-4(d). The rectangular lattice in nanoribbons with $W=3 a$ in Fig. 4(d) represents the distribution of $\pi$ electrons at an energy level close to $\varepsilon_{\mathrm{F}}$ in the Clar representation in Fig. 5(c). See the equivalent Clar formulas of AGNR at the three subclasses in the SM [62], 
(a)

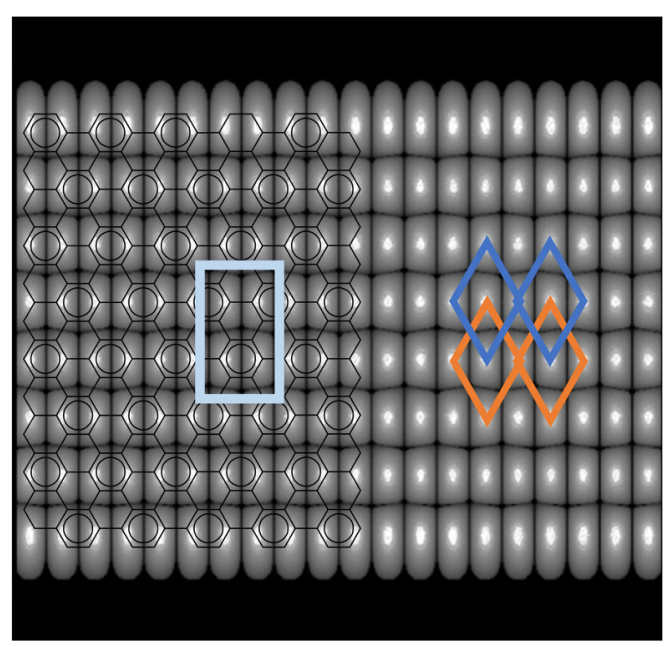

(b)

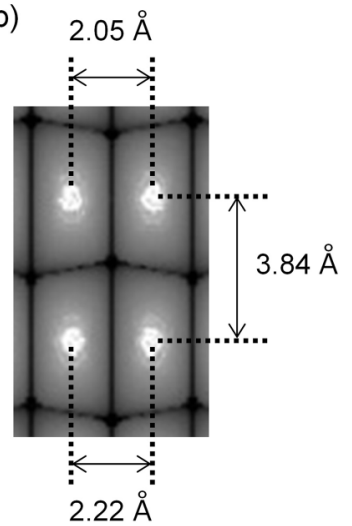

(c)

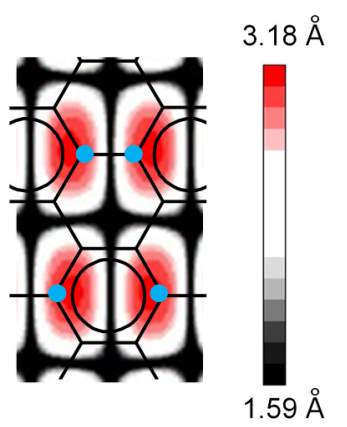

FIG. 6. (a) Simulated STM image of an AGNR with width index $W=24$ at a sample bias of $-0.05 \mathrm{~V}$. The Clar representation is partially superimposed. Orange and blue rhombuses indicate the overlapping of two different $\sqrt{3} \times \sqrt{3}$ phases. (b) Simulated image of the area indicated by the solid line-box in (a). The distances between the bright spots are indicated with arrows. In (a) and (b), a nonlinear gray scale is used to highlight the highest sites at each vertex of the quadrangle lattice. (c) The area in (b) is depicted using a linear colored scale with the corresponding Clar formula. Filled (blue) circles represent the positions of the nearest carbon atoms from the sites with high density of states. These carbon atoms form a trapezoid whereas the four red ovals are the vertices of the rectangularlike lattice.

which also displays calculated STM images at different $V_{\mathrm{s}}$ to compare with those reported by Wassmann et al. [66].

We now look more carefully into the dot patterns of the rectangular lattice. Figure 6(a) is modified from Fig. 4(d) with a partial superimposition of Clar formula. The simulated corrugation in the area surrounded by the solid line is enlarged in Fig. 6(b). The atomic coordinate of the highest points reveal that the four vertices do not form a perfect rectangle but form a trapezoid lattice, as shown in Fig. 6(b). This means that not all four vertices are equivalent sites, i.e., this rectangularlike lattice in Fig. 6(b) can be interpreted as the overlapping of two different $\sqrt{3} \times \sqrt{3}$ phases as shown by the orange and blue rhombuses in Figs. 6(a) and S4 in the SM. However, the difference in length between the upper $(2.05 \AA)$ and lower $(2.22 \AA)$ bases in Fig. 6(b) is very small. Such a subtle difference may be difficult to distinguish clearly in STM experiments. Therefore, the rectangular lattices in the hexagonal graphene network were imaged. The superimposed Clar representation in Fig. 6(c) indicates that the two horizontal bonds in a Clar sextet and the bonds sticking out of the sextet both appear dark. In contrast, side areas in a Clar sextet including oblique bonds appear bright (red), reflecting the high local density of occupied $\pi$ states. In each bright (red) area, the highest corrugation is not at the position of a carbon atom but is situated slightly inside a Clar sextet. As presented in Fig. 6(b), this explains the appearance of the rectangular lattice in STM experiments. As shown in Fig. S3 in the SM, a simulated STM image at a higher sample bias, or a negative bias with a larger absolute value than that in Fig. 4, did not show this rectangular lattice. Consequently, electrons at high energy levels in the occupied states likely contribute to its imaging, some candidates of which are at the HOMO orbital and in the first HOMO band. These details will be discussed elsewhere. In addition, it should be noted that the high-corrugation area or the red area forming the vertex of a rectangular lattice exhibits an oval shape rather than a circular shape in Fig. 6(c). This feature also agrees well with Fig. 2(g), in which each bright dot is elongated along the long-side direction of the rectangle.

We have shown that two armchair edges belonging to AGNR with $W=3 a$ create the unique rectangularlike pattern of electronic structures near $\varepsilon_{\mathrm{F}}$, which resembles those observed experimentally in Fig. 2(g). However, a further point to discuss is the reason why our STM images exhibited a rectangular lattice on the small graphene sheet that is not a simple nanoribbon with perfect armchair edges. Three areas in Fig. 2(d) were extracted to investigate further, shown in Fig. 7(a). Figure 7(b) summarizes average cross-sectional profiles in the rectangular dashed areas shown in Fig. 7(a), projected in the direction of the arrows. The three areas in Fig. 7(a) are enlarged in Figs. 7(c)-7(e). In Fig. 7(c), the flat area consists of a rhombus lattice, or a $\sqrt{3} \times \sqrt{3}$ phase, as indicated by the superimposed unit cell. In Figs. 7(d) and 7(e), an area with rectangular lattices is beside the $\sqrt{3} \times \sqrt{3}$ phase and the boundary is likely to be situated between the two triangular markers in each image. According to Fig. 7(b), height differences of $\sim 0.02 \mathrm{~nm}$ and $>0.1 \mathrm{~nm}$ exist across the boundaries in Figs. 7(d) and 7(e), respectively. A likely explanation of this height difference is the wrinkling of the graphene sheet.

We believe that the area with rectangular lattices is partitioned by two boundaries along the direction perpendicular to the long side of the rectangle. As explained above, one boundary is the wrinkling of the sheet in both Figs. 7(d) and 7(e). Regarding the other boundary of the area with the rectangular lattices in Fig. 7(d), there exists an outer edge of the sheet in the upper-left direction on the outside of Fig. 7(d), as indicated by the orange arrow in Fig. 2(a). And a ridgelike protrusion in the lower area outside of Fig. 7(e) is marked by the green arrow in Fig. 2(a). The height of this protrusion, which may be an adsorbate, was approximately $0.7 \mathrm{~nm}$. Unfortunately, the atomic-scale structures of these defects (the outer edge and the ridgelike structure) were unclear. However, they likely act as the other boundary of the areas with rectangular lattices. 
(a)

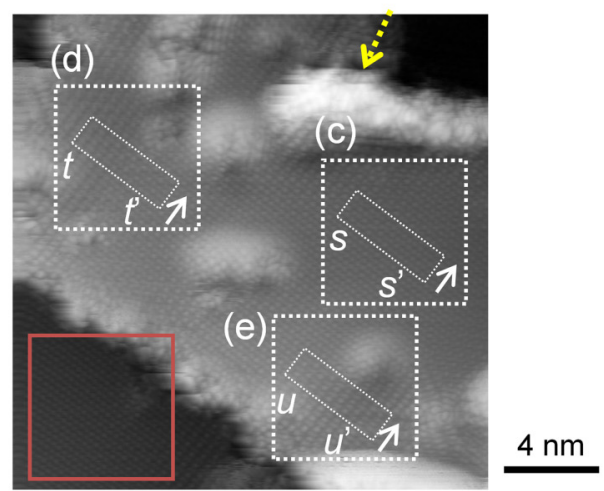

(b)

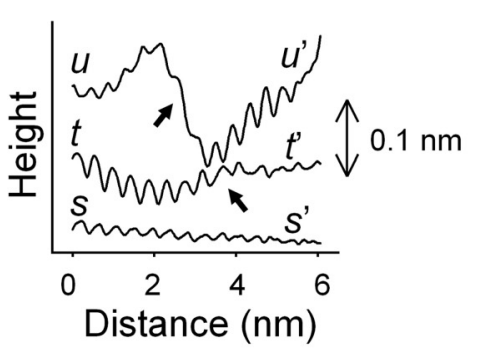

(d)

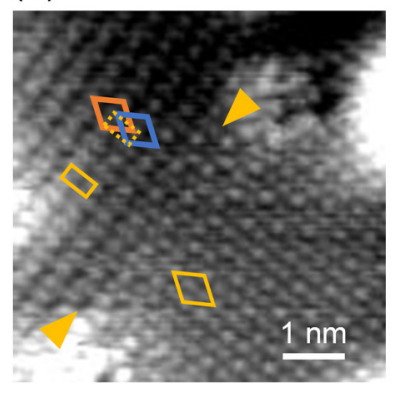

(c)

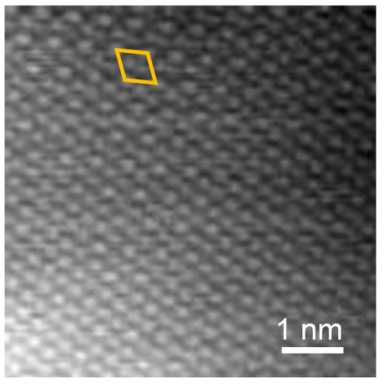

(e)

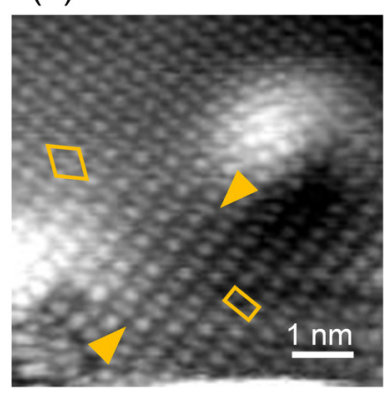

FIG. 7. Analysis of wrinkling of the graphene sheet. (a) Image from Fig. 2(d). The dotted arrow indicates the same position by that in Fig. 2(a). Squares represent four selected areas. (b) Cross-sectional profiles along $s-s^{\prime}, t-t^{\prime}$, and $u-u^{\prime}$ lines in (a). Each profile is an average of the areas indicated by the dashed lines. The profiles are shifted vertically to avoid mixing. In the $t-t^{\prime}$ and $u-u^{\prime}$ lines, there exist height changes, as indicated by arrows. (c)-(e) Enlarged images of the three regions in (a) with enhanced contrast. (c) A relatively flat area with a $\sqrt{3} \times \sqrt{3}$ phase. (d), (e) Regions with a rectangular phase neighboring a $\sqrt{3} \times \sqrt{3}$ area. The approximate position of its boundary in the middle of each image is indicated by filled triangles.

Since first recognized in the $1980 \mathrm{~s}$, the $(\sqrt{3} \times \sqrt{3}) R 30^{\circ}$ phase has appeared not only near a step edge but also near a localized defect or adsorbed molecules on a graphite surface $[29,67]$. More recently, Wang et al. demonstrated AGNRs with atomically well-defined widths in a large graphene sheet on a C-faced $\mathrm{SiC}$ surface. Their AGNRs on nanoterraces were partitioned by pinning regions on the sidewall of the $\mathrm{SiC}$ substrate [60]. In the pinning regions, carbon atoms in graphene strongly bind to the exposed $\mathrm{Si}$ atoms. They suggest a "chemical cutting" enabled realization of AGNRs (a)

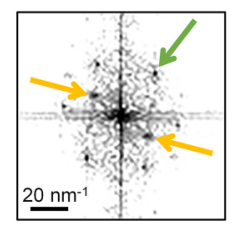

(b)

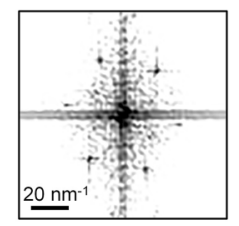

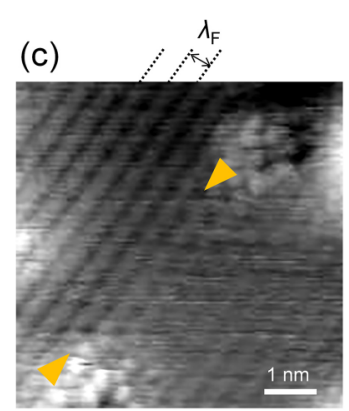

FIG. 8. (a) Two-dimensional fast Fourier transform (2D-FFT) of Fig. 7(d). The green and yellow arrows represent the carbon lattice and $k$ points of the first Brillouin zone, respectively. (b) 2D-FFT image of the area indicated by the square with a solid line in Fig. 7(a). (c) Inverse FFT of (a) masking points from the carbon lattice.

in one large graphene sheet. Considering these studies, it seems reasonable that wrinkling and adsorbates in our folded graphene play a similar role as an armchair edge to generate the $(\sqrt{3} \times \sqrt{3}) R 30^{\circ}$ superstructure. When the separation of these defects is at the nanometer scale, two different $\sqrt{3} \times \sqrt{3}$ phases from them overlap to cause the $\pi$-electron distribution, as shown in Fig. 6. This is an imaging mechanism of the rectangular lattices obtained using STM experiments. The overlap of two $\sqrt{3} \times \sqrt{3}$ phases is schematically depicted as orange and blue rhombuses in Fig. 7(d) as well. Further investigation on the influence of local defects in graphene sheets on the formation of Clar formulas is required. However, the density of wrinkling can be considered higher on a folded or dispersed graphene sheet than on a natural HOPG surface. This may be one of the reasons why the rectangular phase was easily observed on our graphene sheets in Figs. 2 and 3.

In the preceding paragraphs, we discussed the origin of the rectangular lattice based on the resonance or valence-bond theory. Before the conclusion of the paper, we examine it from a different aspect in the scattering of electron waves; we use the STM image in Fig. 7(d), and Fig. 8(a) is its Fourier transform. The outer six points in Fig. 8(a) correspond to the carbon lattice. An example of this is indicated by a green arrow. It also shows two intense points indicated by yellow arrows. They are two of the six possible $k$ points of the first Brillouin zone, indicating the generation of intervalley backscattering processes (i.e., coupling between states at two opposite $K$ points $\left(K\right.$ and $K^{\prime}$ ) [68]). We confirm that the two inner points were weak on the HOPG substrate, as shown in Fig. 8(b). This means that the incident $K\left(K^{\prime}\right)$ and reflected $K^{\prime}$ $(K)$ states cause interference of electron waves, thus resulting in a standing wave state [69] with $(\sqrt{3} \times \sqrt{3}) R 30^{\circ}$ periodicities [36] in Fig. 7(d). Figure 8(c) shows the inverse fast Fourier transform (FFT) when the outer six points from the carbon lattice in Fig. 8(a) are masked. It shows an interference pattern resulting from the two $k$ points indicated by the yellow arrows in Fig. 8(a). This pattern has a periodicity of wavelength $\left(\lambda_{F}\right)$ of $3 a / 2$ [36], where $a=2.46 \AA$. The FFT map in Fig. 8(a) is similar to that on a graphene monolayer with irregular armchair edges on $\mathrm{SiC}$ [70], suggesting that a wrinkle indicated by the triangles in Fig. 8(c) behaves like an armchair edge to cause intervalley backscattering and interference of electron waves. 
We observed in Fig. 8(c) that the upper-left area comprising the rectangularlike lattices has a clearer interference pattern or a stronger standing wave than that in the lower-right area with only the $\sqrt{3} \times \sqrt{3}$ phase. This is because the upper-left area in Fig. 8(c) is partitioned by two boundaries, as mentioned in the previous paragraphs. One is the wrinkle in Fig. 8(c), and the other is a graphene edge indicated by the orange arrow in Fig. 2(a). Generated standing waves from the two boundaries, both having a periodicity of $\left(\lambda_{F}\right)$, coexist in the confined area. This leads to the overlapping of two different $\sqrt{3} \times \sqrt{3}$ phases, forming a rectangularlike lattice, as shown in Figs. 6 and 7(d).

\section{SUMMARY}

Graphite surfaces have been widely used as samples for STM since its invention approximately 40 years ago. In this article, we conducted STM observations of graphene nanosheets on graphite and found a unique rectangular superstructure. Atomic-scale analysis of the images provided insight into the origin of this unique quadrangle lattice, which is an overlap of $\sqrt{3} \times \sqrt{3}$ superstructures along its long diagonals. This was examined by first-principles calculations using a simple model or AGNRs. Based on Clar's theory, it is widely accepted that the electronic structures of AGNRs can be categorized into three subclasses. We found that, with a specific width $(W=3 a)$ among the subclasses, the distribution of electrons with high energy in the occupied band exhibited a rectangularlike lattice in simulated STM images. The shape and distribution of bright dots forming the lattices were consistent with those observed experimentally. From these results, we proposed that (1) wrinkles and other local defects, such as an adsorbate in the graphene sheet, play a similar role as the armchair edge and induce electron wave scattering to form $(\sqrt{3} \times \sqrt{3}) R 30^{\circ}$ superstructures; (2) when these local defects are in close proximity, the region between the defects shows almost the same electronic structures as AGNRs and a rectangular lattice was formed in our small graphene sheet. This study contributes to deeper understanding of electrical and chemical properties of graphene, which can lead to novel applications in technological fields, such as catalytic processes and nanoelectronics.

\section{ACKNOWLEDGMENTS}

The experiments were carried out at the Ultra Clean Room in Osaka University. The computation in this work has been done using the facilities of the Supercomputer Center, the Institute for Solid State Physics, the University of Tokyo. This work was supported by the Japan Society for the Promotion of Science (JSPS) KAKENHI Grant No. JP18K18808. This work was also supported in part by Mitutoyo Association for Science and Technology [Grant No. R2001].
[1] K. S. Novoselov, A. K. Geim, S. V. Morozov, D. Jiang, Y. Zhang, S. V. Dubonos, I. V. Grigorieva, and A. A. Firsov, Science 306, 666 (2004).

[2] Y. Zhang, Y.-W. Tan, H. L. Stormer, and P. Kim, Nature (London) 438, 201 (2005).

[3] A. H. Castro Neto, F. Guinea, N. M. R. Peres, K. S. Novoselov, and A. K. Geim, Rev. Mod. Phys. 81, 109 (2009).

[4] D. C. Elias, R. V. Gorbachev, A. S. Mayorov, S. V. Morozov, A. A. Zhukov, P. Blake, L. A. Ponomarenko, I. V. Grigorieva, K. S. Novoselov, F. Guinea et al., Nat. Phys. 7, 701 (2011).

[5] T. O. Wehling, A. M. Black-Schaffer, and A. V. Balatsky, Adv. Phys. 63, 1 (2014).

[6] M. L. Sadowski, G. Martinez, M. Potemski, C. Berger, and W. A. de Heer, Phys. Rev. Lett. 97, 266405 (2006).

[7] C. W. J. Beenakker, Rev. Mod. Phys. 80, 1337 (2008).

[8] J.-H. Zhong, J. Zhang, X. Jin, J.-Y. Liu, Q. Li, M.-H. Li, W. Cai, D.-Y. Wu, D. Zhan, and B. Ren, J. Am. Chem. Soc. 136, 16609 (2014).

[9] X. Zou and B. I. Yakobson, Acc. Chem. Res. 48, 73 (2015).

[10] X. Yan, Y. Jia, T. Odedairo, X. Zhao, Z. Jin, Z. Zhu, and X. Yao, Chem. Commun. 52, 8156 (2016).

[11] R. Mikurino, A. Ogasawara, T. Hirano, Y. Nakata, H. Yamashita, S. Li, K. Kawai, K. Yamamura, and K. Arima, J. Phys. Chem. C 124, 6121 (2020).

[12] F. Banhart, J. Kotakoski, and A. V. Krasheninnikov, ACS Nano 5, 26 (2011).

[13] S. E. Stein and R. L. Brown, J. Am. Chem. Soc. 109, 3721 (1987).

[14] L. Tapasztó, G. Dobrik, P. Lambin, and L. P. Biró, Nat. Nanotechnol. 3, 397 (2008).
[15] J. Cai, P. Ruffieux, R. Jaafar, M. Bieri, T. Braun, S. Blankenburg, M. Muoth, A. P. Seitsonen, M. Saleh, X. Feng et al., Nature (London) 466, 470 (2010).

[16] P. Ruffieux, S. Wang, B. Yang, C. Sánchez-Sánchez, J. Liu, T. Dienel, L. Talirz, P. Shinde, C. A. Pignedoli, D. Passerone et al., Nature (London) 531, 489 (2016).

[17] L. Brey and H. A. Fertig, Phys. Rev. B 73, 235411 (2006).

[18] T. Wassmann, A. P. Seitsonen, A. M. Saitta, M. Lazzeri, and F. Mauri, Phys. Rev. Lett. 101, 096402 (2008).

[19] C. Casiraghi, A. Hartschuh, H. Qian, S. Piscanec, C. Georgi, A. Fasoli, K. S. Novoselov, D. M. Basko, and A. C. Ferrari, Nano Lett. 9, 1433 (2009).

[20] C. Tao, L. Jiao, O. V. Yazyev, Y.-C. Chen, J. Feng, X. Zhang, R. B. Capaz, J. M. Tour, A. Zettl, S. G. Louie et al., Nat. Phys. 7, 616 (2011).

[21] M. Ziatdinov, S. Fujii, K. Kusakabe, M. Kiguchi, T. Mori, and T. Enoki, Phys. Rev. B 87, 115427 (2013).

[22] K. Kusakabe and M. Maruyama, Phys. Rev. B 67, 092406 (2003).

[23] Y.-W. Son, M. L. Cohen, and S. G. Louie, Nature (London) 444, 347 (2006).

[24] O. V. Yazyev and M. I. Katsnelson, Phys. Rev. Lett. 100, 047209 (2008).

[25] K. Nakada, M. Fujita, G. Dresselhaus, and M. S. Dresselhaus, Phys. Rev. B 54, 17954 (1996).

[26] H. Söde, L. Talirz, O. Gröning, C. A. Pignedoli, R. Berger, X. Feng, K. Müllen, R. Fasel, and P. Ruffieux, Phys. Rev. B 91, 045429 (2015).

[27] X. Zhu and H. Su, J. Phys. Chem. A 115, 11998 (2011). 
[28] M. F. Crommie, C. P. Lutz, and D. M. Eigler, Nature (London) 363, 524 (1993).

[29] J. P. Rabe, M. Sano, D. Batchelder, and A. A. Kalatchev, J. Microsc. 152, 573 (1988).

[30] Y. Kobayashi, K.-I. Fukui, T. Enoki, and K. Kusakabe, Phys. Rev. B 73, 125415 (2006).

[31] W. Zhang, Z. Ju, and W. Wu, Phys. Rev. B 100, 115120 (2019).

[32] P. L. Giunta and S. P. Kelty, J. Chem. Phys. 114, 1807 (2001).

[33] Y. Niimi, T. Matsui, H. Kambara, K. Tagami, M. Tsukada, and H. Fukuyama, Phys. Rev. B 73, 085421 (2006).

[34] K.-I. Sakai, K. Takai, K.-I. Fukui, T. Nakanishi, and T. Enoki, Phys. Rev. B 81, 235417 (2010).

[35] K. A. Ritter and J. W. Lyding, Nat. Mater. 8, 235 (2009).

[36] G. M. Shedd and P. E. Russell, Surf. Sci. 266, 259 (1992).

[37] Ç. Ö. Girit, J. C. Meyer, R. Erni, M. D. Rossell, C. Kisielowski, L. Yang, C.-H. Park, M. F. Crommie, M. L. Cohen, S. G. Louie et al., Science 323, 1705 (2009).

[38] A. Lherbier, S. M.-M. Dubois, X. Declerck, Y.-M. Niquet, S. Roche, and J.-C. Charlier, Phys. Rev. B 86, 075402 (2012).

[39] M. Ziatdinov, S. Fujii, K. Kusakabe, M. Kiguchi, T. Mori, and T. Enoki, Phys. Rev. B 89, 155405 (2014).

[40] T. Hirano, K. Nakade, S. Li, K. Kawai, and K. Arima, Carbon 127, 681 (2018).

[41] S. Li, K. Nakade, T. Hirano, K. Kawai, and K. Arima, Mat. Sci. Semicon. Proc. 87, 32 (2018).

[42] Y. Morikawa, K. Iwata, and K. Terakura, Appl. Surf. Sci. 169-170, 11 (2001).

[43] Y. Morikawa, H. Ishii, and K. Seki, Phys. Rev. B 69, 041403(R) (2004).

[44] J. P. Perdew, K. Burke, and M. Ernzerhof, Phys. Rev. Lett. 77, 3865 (1996).

[45] Y. Baskin and L. Meyer, Phys. Rev. 100, 544 (1955).

[46] J. Tersoff and D. R. Hamann, Phys. Rev. B 31, 805 (1985).

[47] X. Zhang and H. Luo, Appl. Phys. Lett. 103, 231602 (2013).

[48] I. Y. Jumh, B. A. Albiss, and V. V. Dremova, Dig. J. Nanomater. Biostruct. 11, 277 (2016).

[49] V. C. Tung, M. J. Allen, Y. Yang, and R. B. Kaner, Nat. Nanotechnol. 4, 25 (2009).

[50] H. S. Wong, C. Durkan, and N. Chandrasekhar, ACS Nano 3, 3455 (2009).
[51] S. Fujii and T. Enoki, J. Am. Chem. Soc. 132, 10034 (2010).

[52] L. J. Yin, W. X. Wang, K. K. Feng, J.-C. Nie, C. M. Xiong, R.-F. Dou, and D. G. Naugle, Nanoscale 7, 14865 (2015).

[53] S. I. Park and C. F. Quate, Appl. Phys. Lett. 48, 112 (1986).

[54] D. Tománek and S. G. Louie, Phys. Rev. B 37, 8327 (1988).

[55] M. Ye, Y. T. Cui, Y. Nishimura, Y. Yamada, S. Qiao, A. Kimura, M. Nakatake, H. Namatame, and M. Taniguchi, Eur. Phys. J. B 75, 31 (2010).

[56] R. M. Jacobberger, B. Kiraly, M. Fortin-Deschenes, P. L. Levesque, K. M. McElhinny, G. J. Brady, R. Rojas Delgado, S. Singha Roy, A. Mannix, M. G. Lagally et al., Nat. Commun. 6, 8006 (2015).

[57] A. Luican-Mayer, J. E. Barrios-Vargas, J. T. Falkenberg, G. Autès, A. W. Cummings, D. Soriano, G. Li, M. Brandbyge, O. V. Yazyev, S. Roche et al., 2D Mater. 3, 031005 (2016).

[58] D. Pandey, R. Reifenberger, and R. Piner, Surf. Sci. 602, 1607 (2008).

[59] L. Yang, C.-H. Park, Y.-W. Son, M. L. Cohen, and S. G. Louie, Phys. Rev. Lett. 99, 186801 (2007).

[60] W.-X. Wang, M. Zhou, X. Li, S.-Y. Li, X. Wu, W. Duan, and L. He, Phys. Rev. B 93, 241403(R) (2016).

[61] E. Clar, The Aromatic Sextet (Wiley, London, 1972).

[62] See Supplemental Material at http://link.aps.org/supplemental/ 10.1103/PhysRevB.103.245433 for the Clar's theory on graphene nanoribbons.

[63] M. Randić, Chem. Rev. 103, 3449 (2003).

[64] T. M. Krygowski and M. K. Cyrański, Chem. Rev. 101, 1385 (2001).

[65] I. Gutman, Ž. Tomović, K. Müllen, and J. P. Rabe, Chem. Phys. Lett. 397, 412 (2004).

[66] T. Wassmann, A. P. Seitsonen, A. M. Saitta, M. Lazzeri, and F. Mauri, J. Am. Chem. Soc. 132, 3440 (2010).

[67] H. A. Mizes and J. S. Foster, Science 244, 559 (1989).

[68] P. Ruffieux, M. Melle-Franco, O. Gröning, M. Bielmann, F. Zerbetto, and P. Gröning, Phys. Rev. B 71, 153403 (2005).

[69] K. Sasaki, K. Wakabayashi, and T. Enoki, New J. Phys. 12, 083023 (2010).

[70] H. Yang, A. J. Mayne, M. Boucherit, G. Comtet, G. Dujardin, and Y. Kuk, Nano Lett. 10, 943 (2010). 